\title{
Autoimmune Progesterone Dermatitis: A Complex Case
}

Montoro $\mathrm{J}^{1,2}$, Miquel $\mathrm{FJ}^{1,3}$, Quecedo $\mathrm{E}^{3}$, Martínez $\mathrm{M}^{2}$, Orduña $\mathrm{A}^{2}$, Gimeno $\mathrm{E}^{3}$, Valverde $\mathrm{A}^{4}$

${ }^{1}$ Cutaneous Allergy Unit, Hospital Arnau de Vilanova, Valencia, Spain

${ }^{2}$ Allergy Service, Hospital Arnau de Vilanova, Valencia, Spain

${ }^{3}$ Dermatology Service, Hospital Arnau de Vilanova, Valencia, Spain

${ }^{4}$ Department of Human Anatomy and Embryology, Faculty of Medicine and Odontology, University of Valencia, Valencia, Spain

J Investig Allergol Clin Immunol 2018; Vol. 28(5): 348-350 doi: $10.18176 /$ jiaci.0284

Key words: Autoimmune progesterone dermatitis. GnRH analogues. Atopic dermatitis. Food allergy. Lipid transfer protein (LTP).

Palabras clave: Dermatitis autoinmune por progesterona. Análogos de la GnRH. Dermatitis atópica. Alergia alimentaria. Proteína transportadora de lípidos (LTP).

Autoimmune progesterone dermatitis (APD) is an uncommon condition and often a diagnostic challenge. It is characterized by hypersensitivity to progestogens that manifests clinically in a cyclical manner beginning some days before onset of menstruation and resolving during or shortly after the menstrual period.

Clinical presentation is extremely variable, the most common forms being urticaria and eczematous dermatitis. Other manifestations include morbilliform exanthema, bullous eruptions, erythema multiforme, and even purpura [1,2].

The first symptoms can arise as early as menarche, although onset during pregnancy or after hormonal therapy is common.

No specific histopathologic features have been described. Diagnosis is based on the clinical history and confirmed by a positive intradermal test with progesterone.

Therapy usually involves suppression of ovulation using different strategies, including contraceptive pills, GnRH analogues, and danazol. Other options in refractory cases are desensitization [3] and, as a last resort, bilateral oophorectomy.

We report the case of a 50-year-old woman who was diagnosed with atopic dermatitis 12 years ago. She had experienced clinical worsening during the last 2 years and received cyclosporine (200 $\mathrm{mg}$ daily) owing to the failure of previous treatments (topical and oral corticosteroids, bilastine, pimecrolimus, UV-B phototherapy, and off-label omalizumab). Skin biopsy revealed spongiotic dermatitis with no other findings. The patient was referred to our cutaneous allergy unit for patch testing, which yielded negative results with the Spanish baseline series. During history taking, the patient reported that she had been experiencing cyclical flares of her dermatitis over the last 6 months, involving mainly the face and neck (Figure, A). The flares started on days 8-12 of the menstrual cycle and resolved on days 1-2 of menstruation. 


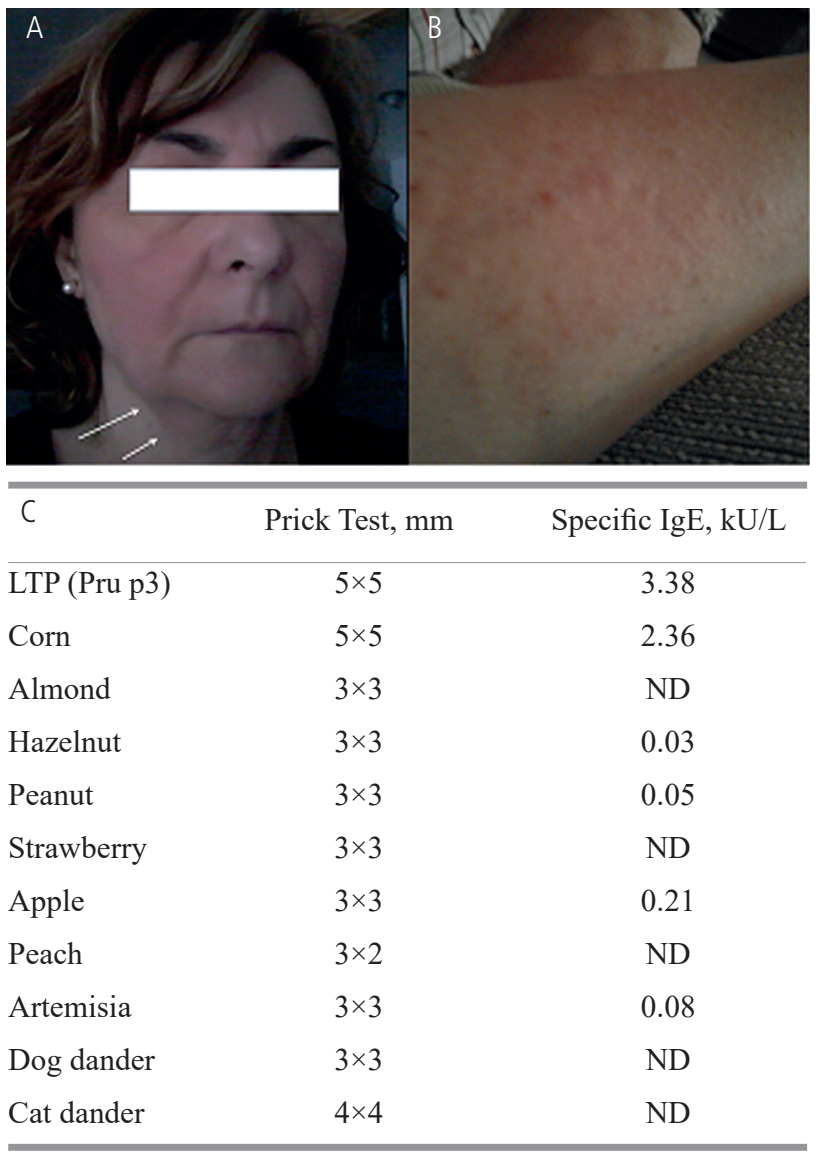

Figure. A, Involvement of the face and neck (arrows). B, Intradermal test with medroxyprogesterone $50 \mathrm{mg} / \mathrm{mL}$. Positive result after 30 hours. C, Positive prick test and specific lgE. ND indicates not done; LTP, lipid transfer protein.

We suspected APD and performed prick and intradermal tests with medroxyprogesterone up to $50 \mathrm{mg} / \mathrm{mL}$ in aqueous solution. The results were negative. This test was repeated 3 weeks after cyclosporine was discontinued, and a late positive reaction ( 30 hours) to the prick and intradermal test was obtained, with erythema and papules that took $>48$ hours to resolve (Figure, B).

After gynecologic assessment, the patient was started on nasal nafarelin, a GnRH analogue (400 $\mu$ g daily). The drug was well tolerated, and the next flare was of much lower intensity.

In parallel, the cyclosporine dose was tapered by $50 \mathrm{mg}$ every other week until complete withdrawal.

Four months later, nasal nafarelin was replaced with triptorelin (monthly 3.75-mg injections), since this was more comfortable for the patient, leaving the first option as rescue medication.

Once the patient started therapy with nafarelin, the dose of oral deflazacort was reduced to only 15-30 mg on 1-2 days per month to manage occasional flare-ups of dermatitis, which at that time were almost controlled with topical methylprednisolone aceponate.

Four months later, atopic dermatitis and APD were well controlled, although the patient developed oral allergy syndrome with recurrent dermatitis outbreaks that were initially related to the ingestion of apple and herb juice.

Prick tests and specific IgE to environmental allergens and foods yielded positive results (Figure, C). Nuts, apple, and peach, which contain lipid transfer protein, were considered clinically relevant. No clinical correlations were observed for the other foods to which the patient was sensitized. Oral symptoms and flare-ups of dermatitis resolved when the culprit foods were avoided, and the lesions of atopic dermatitis were better controlled.

The patient's APD symptoms are now minimal, with welltolerated, faint facial erythema once or twice monthly.

Provided that APD was controlled, and taking into account possible long-term adverse effects, we reduced the dose of triptorelin to $1.87 \mathrm{mg}$ and increased the dosing interval to 6 weeks for 2 additional doses. The patient stopped triptorelin 8 months ago without worsening, even after 4 menstrual cycles, and with detectable serum progesterone levels. Current therapy for atopic dermatitis consists only of emollients and occasional applications of methylprednisolone aceponate cream.

We report a complex and unusual case, in which different forms of allergic disease co-occur, namely, atopic dermatitis, APD, and food allergy.

We infer that the patient had had atopic dermatitis for over 12 years, with significant worsening in the last 2 years, probably related to superimposed APD that could be suspected because she reported cyclical aggravation of her dermatitis during the previous few months. The patient has taken oral contraceptives containing progestagen for dysmenorrhea (between the ages of 18 to 23 years and for some months at age 42). Once APD was controlled, the noncyclical manifestations of atopic dermatitis improved considerably, thus enabling withdrawal of cyclosporine and reducing the need for oral corticosteroids.

The negative result of the first intradermal test with medroxyprogesterone, while the patient was still receiving cyclosporine, along with the positive late reaction observed 3 weeks after stopping the drug, supports the hypothesis that, in this case, the pathogenetic mechanism depends on a type IV, $\mathrm{T}$ cell-mediated hypersensitivity reaction. This contrasts with most previously published cases, in which immediate reactions predominate. We found only 1 report describing a late reaction [4]. It is noteworthy that cyclosporine inhibited the intradermal test, yet it was not able to control APD outbreaks. This observation could be explained by assuming that endogenous progesterone was probably a stronger immunologic stimulus than the concentration of intradermal progestagen.

Suppressing ovulation for 12 months undoubtedly modified the course of APD, with the result that the patient could tolerate endogenous progesterone, as she had normal menstrual cycles after stopping triptorelin.

Type I IgE-mediated hypersensitivity is an aggravating factor in atopic dermatitis. The clinical expertise of the physician is crucial in determining the relevance of allergic sensitizations detected by prick testing or in vitro tests and the potential role that allergen exposure might play in specific cases [5]. In the patient we report, sensitization to 
the panallergen lipid transfer protein could have caused atopic dermatitis before the onset of symptoms of oral allergy syndrome, although with no clear clinical correlation. In any case, avoidance of clinically relevant culprit foods (all of which contain lipid transfer protein) eventually helped to optimize control of atopic dermatitis, thus highlighting the importance of a thorough allergology work-up in difficult-to-control disease, in addition to merely prescribing symptomatic treatment.

\section{Funding}

The authors declare that no funding was received for the present study.

\section{Conflicts of Interest}

The authors declare that they have no conflicts of interest.

\section{References}

1. Grunnet KM, Powell KS, Miller IA, Davis LS. Autoimmune progesterone dermatitis manifesting as mucosal erythema multiforme in the setting of HIV infection. JAAD Case Rep. 2017;3:22-4.

2. Özmen I, Aktürk E. Autoimmune progesterone dermatitis presenting with purpura. Cutis. 2016;98:E12-E13.

3. Foer $D$, Buchheit KM, Gargiulo AP, Lynch DM, Castells $M$, Wickner PG. Progestogen hypersensitivity in 24 cases: diagnosis, management and proposed renaming and classification. J Allergy Clin Immunol Pract. 2016;4:723-9.

4. Frieder J, Younus M. Autoimmune progesterone dermatitis with delayed intradermal skin reaction: A case report. Ann Allergy Asthma Immunol. 2016;117:438-9.

5. Silvestre JF, Romero D, Encabo B. Atopic dermatitis in adults: a diagnostic challenge. J Investig Allergol Clin Immunol. 2017;27:78-88.

Manuscript received April 9, 2018; accepted for publication June 14, 2018.

Javier Montoro Lacomba

Unidad de Alergia Cutánea Servicio de Alergia, Hospital Arnau de Vilanova Calle San Clemente 12 46015 Valencia, Spain E-mail: montoro_fra@gva.es 